TITLE:

\title{
Magnetic anisotropy of the spin-ice compound Dy2Ti2O7
}

\section{$\operatorname{AUTHOR}(\mathrm{S})$ :}

Fukazawa, H; Melko, RG; Higashinaka, R; Maeno, Y; Gingras, MJP

\section{CITATION:}

Fukazawa, H ...[et al]. Magnetic anisotropy of the spin-ice compound Dy2Ti207. PHYSICAL REVIEW B 2002, 65(5): 054410.

\section{ISSUE DATE:}

2002-02-01

URL:

http://hdl.handle.net/2433/49928

RIGHT:

Copyright 2002 American Physical Society 
PHYSICAL REVIEW B, VOLUME 65, 054410

\title{
Magnetic anisotropy of the spin-ice compound $\mathrm{Dy}_{2} \mathrm{Ti}_{2} \mathrm{O}_{7}$
}

\author{
H. Fukazawa* \\ Department of Physics, Kyoto University, Kyoto 606-8502, Japan \\ R. G. Melko \\ Department of Physics, University of Waterloo, Waterloo, Ontario, Canada N2L $3 G 1$
}

R. Higashinaka

Department of Physics, Kyoto University, Kyoto 606-8502, Japan

Y. Maeno

Department of Physics, Kyoto University, Kyoto 606-8502, Japan;

International Innovation Center, Kyoto University, Kyoto 606-8501, Japan;

and CREST, Japan Science and Technology Corporation, Kawaguchi, Saitama 332-0012, Japan

M. J. P. Gingras

Department of Physics, University of Waterloo, Waterloo, Ontario, Canada N2L $3 G 1$

and Canadian Institute for Advanced Research, 180 Dundas Street West, Toronto, Ontario, Canada M5G 1Z8

(Received 8 August 2001; published 8 January 2002)

\begin{abstract}
We report magnetization and ac susceptibility of single crystals of the spin-ice compound $\mathrm{Dy}_{2} \mathrm{Ti}_{2} \mathrm{O}_{7}$. Saturated moments at $1.8 \mathrm{~K}$ along the characteristic axes [100] and [110] agree with the expected values for an effective ferromagnetic nearest-neighbor Ising pyrochlore with local $\langle 111\rangle$ anisotropy, where each magnetic moment is constrained to obey the "ice rule." At high enough magnetic fields along the [111] axis, the saturated moment exhibits a breaking of the ice rule; it agrees with the value expected for a three-in, one-out spin configuration. Assuming the realistic magnetic interaction between Dy ions given by the dipolar spin ice model, we completely reproduce the results at $2 \mathrm{~K}$ by Monte Carlo calculations. However, down to at least 60 $\mathrm{mK}$, we have not found any experimental evidence of the long-range magnetic ordering predicted by this model to occur at around $180 \mathrm{mK}$. Instead, we confirm the spin freezing of the system below $0.5 \mathrm{~K}$.

DOI: 10.1103/PhysRevB.65.054410

PACS number(s): 75.40.Cx, 75.10.Hk, 05.50.+q, 75.40.Mg
\end{abstract}

\section{INTRODUCTION}

The observed absence of magnetic ordering in the Ising pyrochlore magnet $\mathrm{Ho}_{2} \mathrm{Ti}_{2} \mathrm{O}_{7}$ had a strong impact on the present study of frustrated systems. ${ }^{1}$ This compound was known to have a ferromagnetic (FM) Curie-Weiss temperature $\theta_{\mathrm{CW}} \sim 1.9 \mathrm{~K}$, and magnetic $\mathrm{Ho}^{3+}$ ions which possess a strong Ising anisotropy along the local $\langle 111\rangle$ axis. Previous to this discovery, it was expected that absence of ordering would take place in the pyrochlore lattice only with antiferromagnetic Heisenberg spins.,

Harris et al. ${ }^{1}$ showed the possibility of a macroscopically degenerate ground state in $\mathrm{Ho}_{2} \mathrm{Ti}_{2} \mathrm{O}_{7}$ by explaining the multiplicity of the possible spin configurations. By analogy with proton disorder in ice $I_{\mathrm{h}},{ }^{1,4}$ the spin configuration with two spins pointing inward and two spins pointing outward for each tetrahedron corresponds to the "ice rule" for these compounds. Accordingly, $\mathrm{Ho}_{2} \mathrm{Ti}_{2} \mathrm{O}_{7}$ and related compounds such as $\mathrm{Dy}_{2} \mathrm{Ti}_{2} \mathrm{O}_{7}$, which also possess FM Ising spins on a pyrochlore lattice, were named "spin ices.",

The first naive theoretical model of the spin-ice materials placed Ising spins on a pyrochlore lattice, constrained to point along the local $\langle 111\rangle$ direction, with only nearestneighbor FM exchange interactions. ${ }^{1,6}$ However, it was soon deduced that the nearest-neighbor exchange interaction in these compounds is actually antiferromagnetic ${ }^{7}$ and that the effective nearest-neighbor interaction becomes ferromagnetic due to the long-range dipolar interaction arising from the large moments of the $\mathrm{Ho}^{3+}$ and $\mathrm{Dy}^{3+}$ ions. ${ }^{8}$ The physical model which correctly incorporated all of these interactions is called the dipolar spin ice model. Physical quantities (neutron-scattering cross sections and specific-heat curves) calculated from Monte Carlo simulations of this model are in excellent agreement with the corresponding experimental data. $^{8-10}$

Most experimental studies on the spin ices to date have been performed on polycrystalline samples. ${ }^{1,7,9,11-13}$ Among these various experiments, the most dramatic and direct evidence for realization of the spin-ice state is the residual entropy equal to Pauling's entropy: $\frac{1}{2} R \ln \frac{3}{2}=1.68 \mathrm{~J} / \mathrm{K}^{2} \mathrm{~mol}^{4}$ Ramirez et al. obtained an entropy very close to this value $\left[\sim 1.9(2) \mathrm{J} / \mathrm{K}^{2} \mathrm{~mol} / \mathrm{Dy}\right]$ by integrating the experimental electronic specific heat $C_{e} / T$ of $\mathrm{Dy}_{2} \mathrm{Ti}_{2} \mathrm{O}_{7}$ above $0.25 \mathrm{~K}$. This residual entropy can be removed by applying an external magnetic field, since the magnetic field can lift the macroscopic degeneracy by stabilizing a specific spin configuration with lower magnetic energy (Fig. 1), as proven by neutron-diffraction measurement on single crystals of $\mathrm{Ho}_{2} \mathrm{Ti}_{2} \mathrm{O}_{7}$ (Ref. 10) and $\mathrm{Dy}_{2} \mathrm{Ti}_{2} \mathrm{O}_{7}$ (Ref. 14).

Moreover, the electronic specific heat $C_{e}(T) / T$ of $\mathrm{Dy}_{2} \mathrm{Ti}_{2} \mathrm{O}_{7}$ polycrystals exhibit three peaks at $0.34 \mathrm{~K}, 0.47 \mathrm{~K}$, and $1.12 \mathrm{~K}$ independent of the magnitude of the magnetic 
(a)
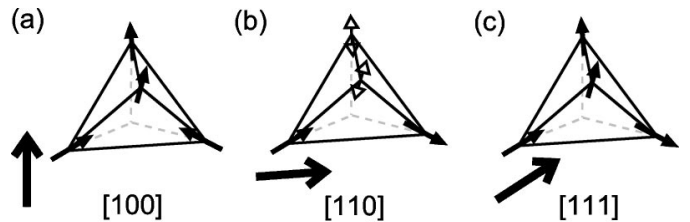

(d)

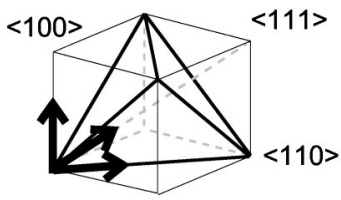

FIG. 1. Spin configurations with the magnetic field along the (a) [100], (b) [110], and (c) [111] axes. For the [111] direction the figure depicts the state in which the ice rule breaks. (d) Threedimensional axes in order to give three-dimensional perspective of the direction in which the field is applied.

field. ${ }^{9}$ Due to the freedom of the decoupled spins of Fig. 1(b), it was speculated by Ramirez et al. that these peaks are attributable to crystal grains with their [110] axis aligned parallel to the field.

The above experimental facts suggest that the spin-ice compounds have significant magnetic anisotopy, and therefore one must control the field direction carefully when studying their physical properties. In particular, the competition between the applied magnetic field and the spin-spin interactions that drive the ice rule obeying degenerate ground state is significantly affected by the crystal orientation in the field. However, to the best of our knowledge, to date there has been no experimental report detailing the behavior of this anisotropy using single crystals of $\mathrm{Dy}_{2} \mathrm{Ti}_{2} \mathrm{O}_{7}$. In this paper, we report such measurements and compare our results with the results predicted by the dipolar spin ice model. We show experimental magnetization results at $1.8 \mathrm{~K}$ along three characteristic axes of this compound, namely, the [100], [110], and [111] axes, and the corresponding numerical results calculated by Monte Carlo simulations. As we will discuss, agreement between the two is excellent. In contrast, a similar set of measurements carried out by Cornelius and Gardner on $\mathrm{Ho}_{2} \mathrm{Ti}_{2} \mathrm{O}_{7}$ failed to find the expected magnetic anisotropy associated with this material. ${ }^{15}$

In addition, it has recently been theoretically predicted that for the dipolar spin ice model and, consequently, for $\mathrm{Dy}_{2} \mathrm{Ti}_{2} \mathrm{O}_{7}$ and $\mathrm{Ho}_{2} \mathrm{Ti}_{2} \mathrm{O}_{7}$, a first-order phase transition should occur at around $180 \mathrm{mK}\left(\equiv T_{\mathrm{c}}\right)$ which fully releases Pauling's ice entropy. ${ }^{16}$ Melko et al. predicted the transition by considering spin loop moves instead of single spin flips within their Monte Carlo simulation, as illustrated in Fig. 1 of Ref. 16. However, few explicit experimental searches for this transition have been reported. ${ }^{14,17}$ Hence, the second motivation for this paper is to investigate single crystals of $\mathrm{Dy}_{2} \mathrm{Ti}_{2} \mathrm{O}_{7}$ using susceptibility measurements down to 60 $\mathrm{mK}$, in order to search for the predicted magnetic ordering. It is hoped that this experimental and theoretical joint study on $\mathrm{Dy}_{2} \mathrm{Ti}_{2} \mathrm{O}_{7}$ can help answer some current issues of debate, and help develop deeper understanding of the nature of the spin-ice materials.

\section{EXPERIMENTS AND CALCULATIONS}

Single crystals of $\mathrm{Dy}_{2} \mathrm{Ti}_{2} \mathrm{O}_{7}$ were grown by the floatingzone method with an infrared furnace equipped with doubleelliptical mirrors. ${ }^{18}$ Before the single-crystal growth we prepared a polycrystalline feed rod by thoroughly mixing $\mathrm{Dy}_{2} \mathrm{O}_{3}(99.99 \%)$ and $\mathrm{TiO}_{2}(99.99 \%)$ powders with the molar ratio of 1 to 2 and sintering the mixture in an alumina crucible at $1000^{\circ} \mathrm{C}$ for $100 \mathrm{~h}$ in air. The typical growth conditions are $29.0 \mathrm{~mm} / \mathrm{h}$ for the feed speed and $30.0 \mathrm{~mm} / \mathrm{h}$ for the crystal-growth speed. To avoid oxygen deficiency, we grew the single crystals in $0.3 \mathrm{MPa}$ of $\mathrm{O}_{2}$ atmosphere. In addition, we annealed the as-grown crystals at $800^{\circ} \mathrm{C}$ for $100 \mathrm{~h}$ in $\mathrm{O}_{2}$ gas flow. The color of the annealed crystals is homogeneously translucent yellow, while that of the asgrown crystals is lighter yellow with some inhomogeneous color distribution. The sizes of samples used for magnetization and ac susceptibility measurements are $0.7 \times 0.7$ $\times 2.0 \mathrm{~mm}^{3}$ and $3.0 \times 1.0 \times 0.2 \mathrm{~mm}^{3}$, respectively. By performing powder $\mathrm{x}$-ray-diffraction measurements on the annealed crystals, we found that the scattering pattern was consistent with a cubic unit cell with $a=10.124(5) \AA$ without any extra peaks. We determined the principal axes using Laue pictures.

We measured magnetization between 1.8 and $40 \mathrm{~K}$ for fields up to $5 \mathrm{~T}$ with a superconducting quantum interference device magnetometer. ${ }^{19}$ We measured ac susceptibility by a mutual-inductance method down to $60 \mathrm{mK}$ using a dilution refrigerator. $^{20}$

For magnetization measurements, we applied the magnetic field in the plane perpendicular to the longest side of the crystal, because all of the principal axes are contained within this plane. The field misalignment is estimated to be within a few degrees. ${ }^{19}$ Thus, we estimate the demagnetization factor $N$ as 0.4 for this crystal. The demagnetization effect can be neglected for ac susceptibility measurements, since the ac field was applied parallel to the longest side, which is parallel to the [100] crystal axis.

In addition, we performed Monte Carlo simulations for magnetization at finite temperatures on the dipolar spin ice model. The detailed calculation techniques are described in Ref. 8. We adopted the following Hamiltonian:

$$
\begin{aligned}
H= & -J \sum_{\langle i, j\rangle} \boldsymbol{S}_{i}^{z_{i}} \cdot \boldsymbol{S}_{j}^{z_{j}}-\sum_{i} \boldsymbol{H}_{\mathrm{eff}} \cdot \boldsymbol{S}_{i}^{z_{i}} \\
& +D r_{\mathrm{nn}}^{3} \sum_{j>i}\left[\frac{\boldsymbol{S}_{i}^{z_{i}} \cdot \boldsymbol{S}_{j}^{z_{j}}}{\left|\boldsymbol{r}_{i j}\right|^{3}}-\frac{3\left(\boldsymbol{S}_{i}^{z_{i}} \cdot \boldsymbol{r}_{i j}\right)\left(\boldsymbol{S}_{j}^{z_{j}} \cdot \boldsymbol{r}_{i j}\right)}{\left|\boldsymbol{r}_{i j}\right|^{5}}\right] .
\end{aligned}
$$

Here $S_{i}^{z_{i}}$ is the Ising moment vector of magnitude of unity at site $i$ pointing along the local Ising axis $z_{i}$. The quantity $\boldsymbol{H}_{\text {eff }}$ is the effective internal magnetic field which directly couples to each spin. For the Hamiltonian coefficients, we used $-1.24 \mathrm{~K}$ for the nearest-neighbor exchange interaction $J_{\mathrm{nn}}$ $\equiv J / 3$ and $2.35 \mathrm{~K}$ for the nearest-neighbor dipolar interaction $D_{\mathrm{nn}} \equiv 5 \mathrm{D} / 3$. We took into account the long-range nature of the dipolar interactions through the Ewald summation technique. A standard Metropolis algorithm was used with a conventional cubic unit cell for the pyrochlore lattice containing 


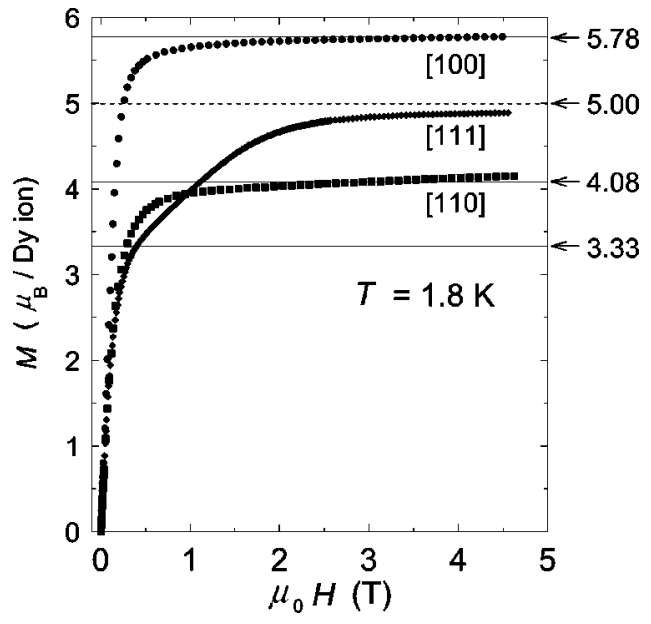

FIG. 2. Magnetization curves at $1.8 \mathrm{~K}$ along the [100], [110], and [111] axes. Solid lines correspond to the expected saturated moments for the two-in, two-out spin configuration for all the directions: dotted line corresponds to that for the three-in, one-out spin configuration.

16 spins. Simulations of the specific heat obtained using these values are in very good agreement with the experimental specific heat reported for $\mathrm{Dy}_{2} \mathrm{Ti}_{2} \mathrm{O}_{7} .{ }^{8}$ The simulation size was $L=3$ (432 spins). When calculating the magnetization curves, a number of different simulation sizes were tried, and little variation resulted.

\section{RESULTS AND DISCUSSION}

\section{A. Magnetic anisotropy}

In Fig. 2 we summarize the magnetization curves at $1.8 \mathrm{~K}$ along the three characteristic axes. We took the horizontal axis as the effective magnetic field, $\mu_{0} H_{\text {eff }}=\mu_{0} H_{\text {ext }}$ $-N M_{\text {exp }}$. We obtained nearly the same data at $2 \mathrm{~K}$ (not shown). We note that no hysteresis is observed between the upward and downward field sweeps.

Saturated moments along the [100] and [110] axes agree with the expected values for Ising anisotropy along the local 〈111〉 direction and for effective FM coupling between the nearest-neighbor Dy ions. The value for the [100] axis is $g_{J} J(1 / \sqrt{3})=5.77 \mu_{\mathrm{B}} /$ Dy [two spins point in and two spins point out: Fig. 1(a)] and that for the [110] axis is $g_{J} J\{(\sqrt{2 / 3} \times 2) / 4\}=4.08 \mu_{\mathrm{B}} /$ Dy [one spin points in, one spin points out, and two spins are free: Fig. 1(b)]. Here, $g_{J}$ represents Landé's $g$ factor, $J$ corresponds to the total angular moment of the $\mathrm{Dy}^{3+}$ ion, and $\mu_{\mathrm{B}}$ represents the Bohr magneton. For the free $\mathrm{Dy}^{3+}$ ion, $g_{J} J$ is expected to be $10 \mu_{\mathrm{B}} / \mathrm{Dy}$. Slight deviation from the expected values may be due to a misalignment effect as we discuss below.

Below $0.5 \mathrm{~T}$, the magnetization along the [111] axis already reaches the value $g_{J} J\{(1+1 / 3 \times 1) / 4\}=3.33 \mu_{\mathrm{B}} / \mathrm{Dy}$ expected for the saturated state in which the ice rule completely holds. In this state, three of the spins per tetrahedra are coupled to minimize their energy with the applied magnetic field along the [111] direction, but one spin chooses to oppose the field and minimize its energy with respect to the effective nearest-neighbor interaction, remaining in the ice

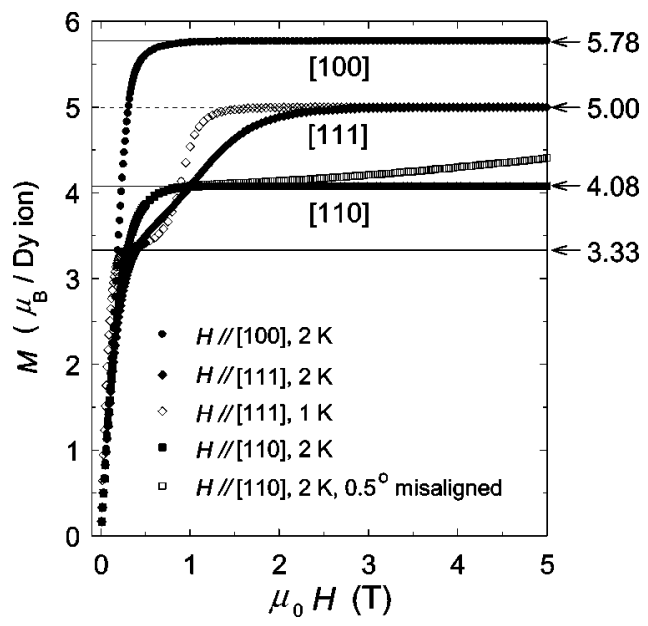

FIG. 3. Magnetization curves calculated by Monte Carlo simulations of the dipolar spin ice model. The simulation size was $L$ $=3$ (432 spins), and parameters were chosen for $\mathrm{Dy}_{2} \mathrm{Ti}_{2} \mathrm{O}_{7}$.

rule. For quite large fields $\left(H_{\mathrm{eff}} \gg J_{\mathrm{nn}}+D_{\mathrm{nn}}\right)$, this configuration begins to cost a large amount of energy, and the fieldopposed spin flips to minimize its energy with respect to the applied magnetic field, hence breaking the ice rule. Accordingly, the magnetization agrees with the value expected for the three-in, one-out spin configuration [Fig. 1(c)], $g_{J} J\{(1$ $+1 / 3 \times 3) / 4\}=5.00 \mu_{\mathrm{B}} / \mathrm{Dy}$. We infer that the gain of Zeeman energy exceeds that of the effective nearest-neighbor FM interaction above $0.5 \mathrm{~T}$ : $(1 / 3) g_{J} J \mu_{\mathrm{B}}\left(\mu_{0} H_{\mathrm{eff}}\right)=1.12 \mathrm{~K}$, in good accordance with $J_{\text {eff }} \equiv J_{\mathrm{nn}}+D_{\mathrm{nn}}=1.11 \mathrm{~K}$.

For fields up to $0.5 \mathrm{~T}$, the moments along all the axes nearly reach the expected values for the corresponding spinice configurations in a magnetic field. This experimental fact indicates that the magnetic anisotropy in $\mathrm{Dy}_{2} \mathrm{Ti}_{2} \mathrm{O}_{7}$ is indeed compatible with the spin-ice model. In addition, the breaking of the ice rule observed along the [111] axis confirms that the strong Ising anisotropy along the local $\langle 111\rangle$ direction dominates in this compound.

A corresponding field dependence of magnetization is reported by Cornelius and Gardner for single crystals of $\mathrm{Ho}_{2} \mathrm{Ti}_{2} \mathrm{O}_{7} \cdot{ }^{15}$ However, their data above $2 \mathrm{~T}$ is totally unexpected, because the magnetizations along all the directions reach approximately $5.9 \mu_{\mathrm{B}} /$ Ho. Their behavior cannot be explained solely by a crystal misalignment effect, since a similar effect of misalignment must also exist to some extent in our experiment, but clearly results in a different behavior than that observed here and in Ref. 21.

In Fig. 3 we show magnetizations at 1 and $2 \mathrm{~K}$ calculated by the Monte Carlo simulations. Each solid symbol represents the magnetization along the corresponding field direction of the experimental results. The open squares are for the magnetization at $2 \mathrm{~K}$ along the [110] axis with intentional $0.5^{\circ}$ misalignment toward the [100] axis. The open diamonds are for the magnetization at $1 \mathrm{~K}$ along the [111] axis.

Clearly the Monte Carlo simulations at $2 \mathrm{~K}$ reproduce our corresponding experimental magnetization curves very well, though the absolute values do not completely coincide with each other. ${ }^{22}$ This excellent agreement between experiments and calculations implies that one may treat this compound as 


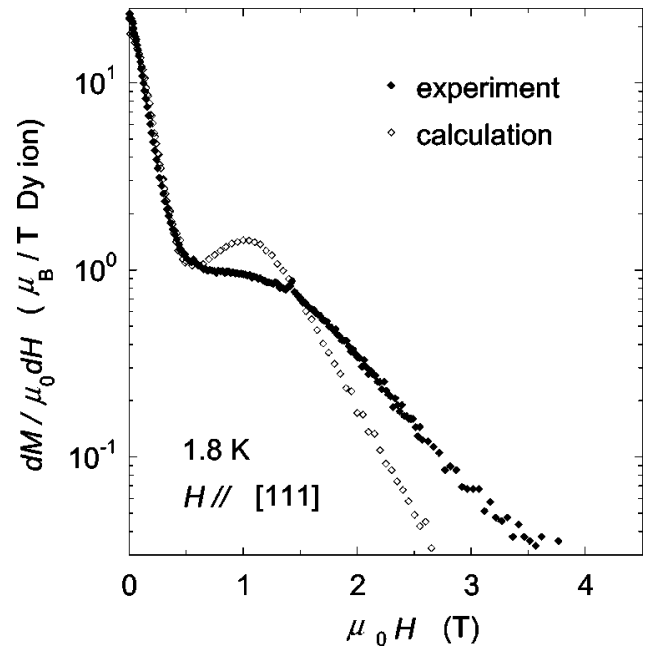

FIG. 4. Derivative of the experimental and calculated magnetizations at $1.8 \mathrm{~K}$ with $\mu_{0} H_{\text {eff }}$ along the [111] axis.

an ideal model system for the study of geometrical frustration in the pyrochlore lattice. Moreover, it shows that the assumption of an Ising doublet with leading wave functions $\left|\psi_{+}\right\rangle$and $\left|\psi_{-}\right\rangle$with $\left|m_{J}\right\rangle$ decompositions $\left|\psi_{ \pm}\right\rangle \approx \mid m_{J}=$ $\pm 15 / 2\rangle$ is a very good approximation, in good agreement with crystal-field calculations for $\mathrm{Dy}_{2} \mathrm{Ti}_{2} \mathrm{O}_{7}$ (Ref. 23) adapted from the crystal-field parameters extracted for $\mathrm{Ho}_{2} \mathrm{Ti}_{2} \mathrm{O}_{7} \cdot{ }^{24}$

The calculated magnetization with the field along the [110] axis with the intentional $0.5^{\circ}$ misalignment deviates substantially from the aligned value at higher fields. This is due to the acute angular sensitivity specific to field application along the [110] axis, since slight deviation of the field from the [110] axis can fix the spin directions of the two free spins normal to the [110] axis in every tetrahedron [Fig. 1(b)]. The increase in magnetization is attributable to the field-coupled component of these spins still satisfying the ice rule. Therefore, the experimental deviation from the expected value, $4.08 \mu_{\mathrm{B}} / \mathrm{Dy}$, can be explained by assuming a crystal misalignment within $0.5^{\circ}$ from the [110] axis.

The derivative of the experimental and calculated magnetizations at $1.8 \mathrm{~K}$ with $H_{\text {eff }}$ along the [111] axis is shown in Fig. 4. The derivative of the experimental magnetization, $d M_{\text {exp }} / d H_{\text {eff }}$, exhibits a definite kink at around $0.5 \mathrm{~T}$ and quite a broad peak at around $1 \mathrm{~T}$. This kink signals the crossover from two-in, two-out spin-ice configuration to three-in, one-out configuration. The derivative of the calculated magnetization, $d M_{\text {cal }} / d H_{\text {eff }}$, also exhibits a local minimum at around $0.5 \mathrm{~T}$ and a broad peak at around $1 \mathrm{~T}$. In addition, the behavior below $0.5 \mathrm{~T}$ nearly coincides with the experimental results. This good agreement between experiments and calculations indicates that the behavior of the dipolar spin ice model in the spin-interacting regime (i.e., at low fields) behaves almost the same as the real materials in the same regime. The difference between them is most likely due to the experimental misalignment effect, since the difference became smaller when we measured another single crystal by adjusting the alignment more accurately. ${ }^{25}$

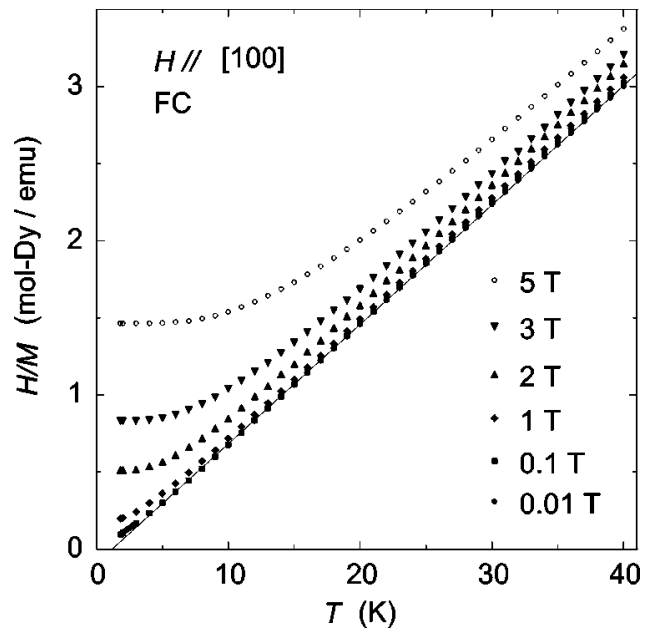

FIG. 5. Temperature dependence of reciprocal dc susceptibility for various magnitudes of magnetic field along the [100] axis.

The calculated broad peak becomes sharper at $1 \mathrm{~K}$ and correspondingly a clear plateau between 0.25 and $0.45 \mathrm{~T}$ emerges in the magnetization curve at $1 \mathrm{~K}$. The plateau definitely suggests that an ice rule obeying spin configuration is more stable than the three-in, one-out spin configuration at lower temperatures and at lower fields. Such a plateau is also reported in $\mathrm{Ho}_{2} \mathrm{Ti}_{2} \mathrm{O}_{7}$ at $1.8 \mathrm{~K}$ between 0.4 and $1.6 \mathrm{~T} .{ }^{15} \mathrm{In}$ $\mathrm{Ho}_{2} \mathrm{Ti}_{2} \mathrm{O}_{7}$, the appearance of the plateau clearly corresponds to the temperature of the maximum in the specific heat. This characteristic temperature is $1.97 \mathrm{~K}_{\text {in }} \mathrm{Ho}_{2} \mathrm{Ti}_{2} \mathrm{O}_{7},{ }^{10}$ and corresponds to the onset of strong spin-ice correlations in the material. The corresponding characteristic temperature in $\mathrm{Dy}_{2} \mathrm{Ti}_{2} \mathrm{O}_{7}$ is $1.2 \mathrm{~K}$. ${ }^{9}$ The results that a clear plateau appears at $1 \mathrm{~K}$ in our calculations but not above $1.8 \mathrm{~K}$ in our experiments as well as our calculations are reasonable compared with the characteristic temperature for $\mathrm{Dy}_{2} \mathrm{Ti}_{2} \mathrm{O}_{7}$.

In Fig. 5 we show the temperature dependence of reciprocal dc susceptibility for various magnetic fields along the [100] axis. The solid line denotes the Curie-Weiss fitting between 16 and $40 \mathrm{~K}$ for the data at $0.01 \mathrm{~T}$ :

$$
\chi(T)=\frac{N_{\mathrm{A}} p_{\mathrm{eff}}^{2} \mu_{\mathrm{B}}^{2} / 3 k_{\mathrm{B}}}{T-\theta_{\mathrm{CW}}}
$$

where $\chi \equiv M / H$ is magnetic susceptibility, $N_{\mathrm{A}}$ is the Avogadro number, $p_{\text {eff }}$ is the effective Bohr magneton, and $k_{\mathrm{B}}$ is the Boltzmann constant. Again, $H$ has been corrected for the demagnetization factor.

For a given temperature, the ratio $H / M$ increases with an increasing magnetic field. This is because the magnetization at higher fields saturates compared with that at lower fields especially at lower temperatures.

We obtained positive Curie-Weiss temperatures 1.16(5), $1.31(5)$, and $1.28(5) \mathrm{K}$ from the data at $0.01 \mathrm{~T}$ along the [100], [110], and [111] axes, respectively. This is consistent with the existence of the effective nearest-neighbor FM interaction, mainly originating from large dipolar interaction between Dy ions, and consistent with the previous results using polycrystalline samples. ${ }^{9,13}$ Moreover, this provides 


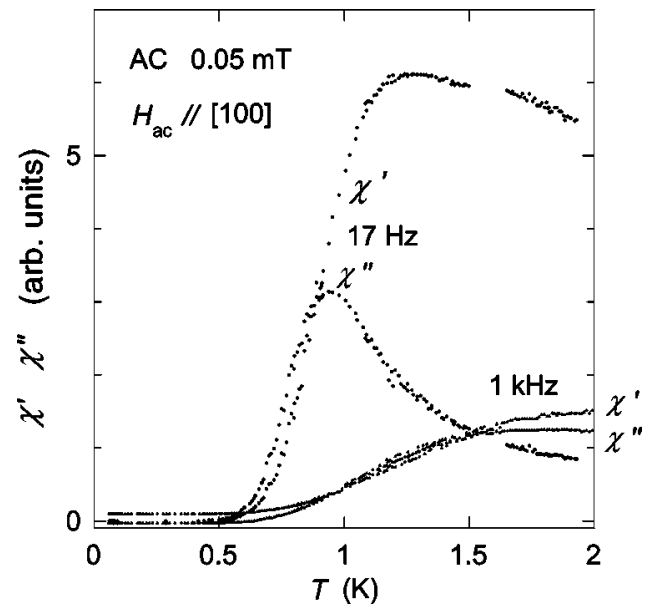

FIG. 6. Ac susceptibility measured down to $60 \mathrm{mK}$.

further validity in using the value for the effective FM interaction used in our calculations, $J_{\text {eff }} \equiv J_{\mathrm{nn}}+D_{\mathrm{nn}}=1.11 \mathrm{~K}^{26}$

We also obtained the effective Bohr magneton 10.2(1), 10.0(1), and 10.0(1) $\mu_{\mathrm{B}} / \mathrm{Dy}$ for the [100], [110], and [111] axes, respectively, from the Curie-Weiss fitting. These values are nearly equal to the value $p_{\text {eff }} \equiv g_{J} \sqrt{J(J+1)}$ $=10.6 \mu_{\mathrm{B}} /$ Dy expected for the completely localized spin of the $\mathrm{Dy}^{3+}$ ion.

\section{B. Ac susceptibility and search for the $180 \mathrm{mK}$ transition}

Considering the above excellent agreement between our experiments and calculations, we may expect the realization of the theoretically predicted first-order phase transition in the real material. ${ }^{16}$ In Fig. 6, we show the ac susceptibility $\chi_{\text {ac }}$ down to $60 \mathrm{mK}$. In order to keep the sample in zero dc field, we surrounded the sample coils with a highpermeability paramagnetic tube. An ac field of $0.05 \mathrm{mT}$ was applied along the [100] axis. This amplitude satisfies the condition, $H_{\mathrm{ext}} / D_{\mathrm{nn}} \sim 2 \times 10^{-4} \ll 0.08 \sim T_{\mathrm{c}} / D_{\mathrm{nn}}$, suggested in Ref. 16. We measured $\chi_{\mathrm{ac}}$ at $1 \mathrm{kHz}$ on cooling and at $17 \mathrm{~Hz}$ on warming after keeping the crystal at $60 \mathrm{mK}$ for $1 \mathrm{~h}$. We normalized the data by dividing the raw data by the measuring frequency.

The in-phase component $\chi^{\prime}$ measured at $17 \mathrm{~Hz}$ exhibits a broad peak at around $1.2 \mathrm{~K}$ and steep decrease below this temperature. In addition, it diminishes below about $0.5 \mathrm{~K} . \chi^{\prime}$ at $1 \mathrm{kHz}$ and the out-of-phase, dissipative components $\chi^{\prime \prime}$ at both frequencies also exibit the similar temperature dependence. These data are consistent with those obtained from polycrystalline $\mathrm{Dy}_{2} \mathrm{Ti}_{2} \mathrm{O}_{7}{ }^{17}$

The absence of any change in the susceptibilities below $0.5 \mathrm{~K}$ indicates that the spins on each tetrahedron in the system become firmly locked in a random ice-rule configuration at about $0.5 \mathrm{~K}$, below which the specific heat has also essentially vanished as shown in the previous study by Ramirez et al. ${ }^{9}$ Further, our data suggest that the predicted first-order phase transition does not occur, at least within the limited experimental measurement time scale. Because the "freeze-in" temperature is well above the predicted ordering temperature of $0.18 \mathrm{~K}$, it is plausible that there is insufficient local spin dynamics left in the system that can promote the ordering associated with the nonlocal dynamics of a spin loop move in the Monte Carlo simulations of Ref. 16. We therefore speculate that the system has essentially completely fallen out of equilibrium at around $0.5 \mathrm{~K}$; below this temperature one does not expect to recover equilibrium in a time that is reasonably accessible by experiment.

The temperatures of the maxima in $\chi^{\prime}$ and $\chi^{\prime \prime}$ increase with increasing frequencies. The dependence is ascribable to a long magnetic relaxation time of the spin lock-in formation as Matsuhira et al. discussed in their previous polycrystalline study. ${ }^{17}$ This also supports our interpretation of the absence of ordering down to $60 \mathrm{mK}$. In further studies, it may be possible that slight dilution of Dy with nonmagnetic elements such as Y may bring the static ordering, in analogy to the transition in water ice from the $I_{\mathrm{h}}$ phase to the long-range ordered crystal $I_{\text {XI }}$ phase via substitution with $\mathrm{KOH}^{27}$

\section{CONCLUSION}

In conclusion, we reported magnetization and ac susceptibility of $\mathrm{Dy}_{2} \mathrm{Ti}_{2} \mathrm{O}_{7}$ using single crystals. The clear magnetic anisotropy indicates the strong Ising anisotropy and the effective FM interaction between the nearest-neighbor Dy ions, as well as the dictating ice rule at low temperatures. These effects have been quantitatively confirmed by both experimental and theoretical approaches in the present study.

No indication of the predicted long-range magnetic ordering was observed. This may be due to differences in time scale between the dynamics of the real system and the simulation. Futher investigations, such as specific-heat measurements, or dilution with nonmagnetic impurities, may clarify the attainability of the real ground state of $\mathrm{Dy}_{2} \mathrm{Ti}_{2} \mathrm{O}_{7}$.

Note added in proof. Interestingly, it has recently been reported that the freezing process in $\mathrm{Dy}_{2} \mathrm{Ti}_{2} \mathrm{O}_{7}$ may also display a signature in the $10-20 \mathrm{~K}$ temperature range. ${ }^{17,28}$

\section{ACKNOWLEDGMENTS}

We acknowledge useful discussions and technical support from D. Yanagishima and H. Yaguchi. We would also like to thank T. Ishiguro for his support in many aspects. We appreciate fruitful discussions with K. Matsuhira, S. Rosenkranz, and Y. Yasui. Finally, we acknowledge B. den Hertog for his contribution to this work. H.F. is grateful for the financial support from JSPS Research Fellowships for Young Scientists. M.G. acknowledges financial support from NSERC of Canada, Research Corporation, and the Province of Ontario.
*Corresponding author. Email address: hideto@scphys.kyotou.ac.jp

${ }^{1}$ M.J. Harris, S.T. Bramwell, D.F. McMorrow, T. Zeiske , and K.W. Godfrey, Phys. Rev. Lett. 79, 2554 (1997).
${ }^{2}$ J.N. Reimers, Phys. Rev. B 45, 7287 (1992); R. Moessner and J.T. Chalker, Phys. Rev. B 58, 12049 (1998).

${ }^{3}$ B. Canals and C. Lacroix, Phys. Rev. Lett. 80, 2933 (1998).

${ }^{4}$ L. Pauling, The Nature of the Chemical Bond (Cornell University 
Press, Ithaca, 1945), p. 301.

${ }^{5}$ For a recent review on spin ice systems, see S.T. Bramwell and M.J.P. Gingras, Science 294, 1495 (2001).

${ }^{6}$ M.J. Harris, S.T. Bramwell, P.C.W. Holdsworth, and J.D.M. Champion, Phys. Rev. Lett. 81, 4496 (1998).

${ }^{7}$ R. Siddharthan, B.S. Shastry, A.P. Ramirez, A. Hayashi, R.J. Cava, and S. Rosenkranz, Phys. Rev. Lett. 83, 1854 (1999).

${ }^{8}$ B.C. den Hertog and M.J.P. Gingras, Phys. Rev. Lett. 84, 3430 (2000).

${ }^{9}$ A.P. Ramirez, A. Hayashi, R.J. Cava, R. Siddharthan, and B.S. Shastry, Nature (London) 399, 333 (1999).

${ }^{10}$ S.T. Bramwell, M.J. Harris, B.C. den Hertog, M.J.P. Gingras, J.S. Gardner, D.F. McMorrow, A.R. Wildes, A. Cornelius, J.D.M. Champion, R.G. Melko, and T. Fennell, Phys. Rev. Lett. 87, 047205 (2001).

${ }^{11}$ Y.M. Jana and D. Ghosh, Phys. Rev. B 61, 9657 (2000).

${ }^{12}$ K. Matsuhira, Y. Hinatsu, K. Tenya, and T. Sakakibara, J. Phys.: Condens. Matter 12, L649 (2000).

${ }^{13}$ S.T. Bramwell, M.N. Field, M.J. Harris., and I.P. Parkin, J. Phys.: Condens. Matter 12, 483 (2000).

${ }^{14}$ T. Fennell, O.A. Petrenko, G. Balakrishnan, S.T. Bramwell, J.D.M. Champion, B. Fak, M.J. Harris, and D.McK. Paul, cond-mat/0107414 (unpublished).

${ }^{15}$ A.L. Cornelius and J.S. Gardner, Phys. Rev. B 64, 060406(R) (2001).

${ }^{16}$ R.G. Melko, B.C. den Hertog, and M.J.P. Gingras, Phys. Rev. Lett. 87, 067203 (2001).

${ }^{17}$ K. Matsuhira, Y. Hinatsu, and T. Sakakibara, J. Phys.: Condens. Matter 13, L737 (2001).

${ }^{18}$ NEC machinery, Model No. SC-E15HD.
${ }^{19}$ Quantum Design, Model No. $\mathrm{MPMS}_{5 \mathrm{~S}}$; we used a single-axis rotator to adjust the field direction.

${ }^{20}$ Oxford Instruments, Kelvinox ${ }^{\text {TLM }}$ model.

${ }^{21}$ Y. Yasui (private communication) showed that their magnetization data of single-crystalline $\mathrm{Ho}_{2} \mathrm{Ti}_{2} \mathrm{O}_{7}$ are quantitavely similar to ours.

${ }^{22}$ Although the difference between the experiment and the calculations at low fields along the [111] direction is small, the difference is evident generally for the lower-field region. This is most likely due to the difficulty in precisely and acurately determining the geometric details of the sample for the demagnetization factor. The difference becomes less than $3 \%$ at $2 \mathrm{~T}$, since it is unimportant to consider the uncertainty of the demagnetization factor at high magnetic fields.

${ }^{23} \mathrm{~S}$. Rosenkranz (private communication).

${ }^{24}$ S. Rosenkranz, A.P. Ramirez, A. Hayashi, R.J. Cava, R. Siddharthan, and B.S. Shastry, J. Appl. Phys. 87, 5914 (2000).

${ }^{25}$ It was quite difficult to exactly align the field direction along the [111] axis with the crystal used. Because all the crystal surfaces used in the magnetization measurements are not parallel to the [111] axis but parallel to the [100] or [110] axes, we could not confirm whether the magnetic field is parallel to the [111] axis by eye.

${ }^{26}$ The determination of the only adjustable parameter $J_{\mathrm{nn}}$ in the dipolar spin ice model was not possible in this study, since the demagnetization factor cannot be estimated exactly. The value of $J_{\text {nn }}$ was taken to be $-1.24 \mathrm{~K}$ from Ref. 8 .

${ }^{27}$ Y. Yamaji, T. Matsuo, and H. Suga, Nature (London) 299, 810 (1982).

${ }^{28}$ J. Snyder, J.S. Slusky, R.J. Cava, and P. Schiffer, Nature (London) 413, 48 (2001). 